\title{
PPI befördern hepatische Enzephalopathie
}

\author{
Bei Patienten mit fortgeschrittener Leberzirrhose sollten Protonenpumpenhemmer (PPI) mit Vorsicht \\ verschrieben werden. Sie könnten das Risiko für eine hepatische Enzephalopathie erhöhen.
}

Wegen ihres vermeintlich guten Sicherheitsprofils werden PPI bei Patienten mit Leberzirrhose großzügig verordnet. Allerdings führen sie zu einer Dysbiose im Gastrointestinaltrakt, was eine bekannte Ursache für die hepatische Enzephalopathie (HE) ist.

Um den Zusammenhang zu untersuchen, wurden die Daten von einer Million Zirrhosepatienten aus der taiwanesischen Krankenversicherungsdatenbank untersucht. Ausgewählt wurden 1.166 Personen, die zwischen 1998 und 2011 erstmalig eine HE erlitten hatten. Als Kontrollgruppe dienten Patienten mit dem gleichen Zirrhosegrad, bei denen keine HE auftrat.
Die Datenbank-Analyse zeigte eine signifikant längere PPI-Einnahme der HE-Patienten (173,7 vs. 130,8 Tage). In der Subgruppenanalyse zeigte sich eine dosisabhängige Korrelation, auch nach der Berücksichtigung anderer Medikamente, Komorbiditäten und demografischer Faktoren. Bei Patienten, die > 365 Tagesdosen PPI einnahmen, war das HE-Risiko dreifach erhöht, bei 121-365 Tagesdosen 1,5-fach und bei 31-120 Tagesdosen 1,4-fach.

Die Korrelation ergab sich für Pantoprazol, Omeprazol, Esomeprazol und Lanzoprazol - nicht aber für Rabeprazol. Dieser PPI hat einen anderen Abbauweg als die anderen Präparate.
- Chia-Fen Tet al. Proton pump inhibitors increase risk for hepatic encephalopathy in patients with cirrhosis in a population study. Gastroenterology. 2017;152:134-41

\section{KOMMENTAR}

Diese Ergebnisse müssen Gastroenterologen und Hausärzte alarmieren, den Einsatz von PPI bei dieser Patientengruppe immer wieder zu hinterfragen. Aktuell erhalten 12-67\% der Leberzirrhosepatienten diese Mittel. Ergibt sich keine eindeutige Indikation für die Einnahme, sollte diese beendet werden. Ist eine Langzeitanwendung notwendig, zeigt Rabeprazol eventuell bessere Ergebnisse und sollte aus der Gruppe der PPI ausgewählt werden.

PD Dr. med. C. Waggershauser

\section{SIPE - der Tod im kalten Wasser}

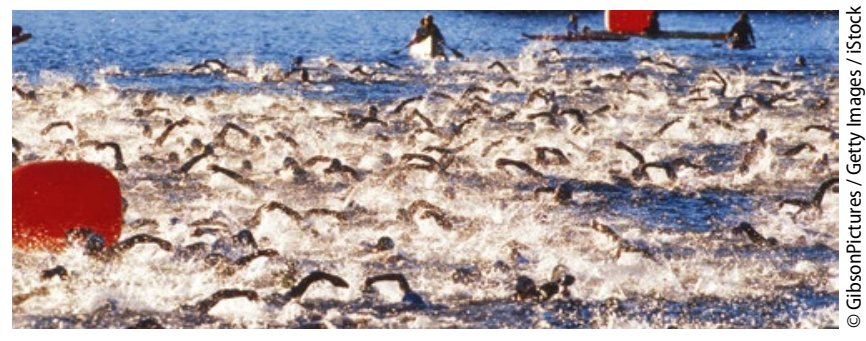

Erwischt es einen dieser Triathleten?

Immer wieder bekommen Triathleten während des Schwimmens eine plötzliche Lungenfunktionsstörung. 2015 starb einer dieser Extremsportler im Hudson River. Eine andere Teilnehmerin entwickelte schwere Atemnot nach einer Hustenattacke. Sie spuckte Blut. Innerhalb kurzer Zeit hatte sich ein Lungenödem entwickelt. Bei beiden wurde ein Swimming-induced pulmonary edema (SIPE) vermutet. Die American Heart Association berichtet von 43 Todesfällen beim Triathlon in den Jahren 2003-2011, davon 30 beim Schwimmen. Das Tückische ist, dass sich die Dyspnoe innerhalb weniger Minuten entwickeln kann.

Die Pathophysiologie des Syndroms ist nicht vollständig geklärt. Sicher spielen die Zunahme des Pulmonalarteriendrucks und ein Stabilitätsverlust der Kapillarwand mit Wassereintritt in die Al- veolen eine wichtige Rolle. Risikokonditionen sind kaltes Wasser, massive körperliche Belastung, erhöhte Flüssigkeitsaufnahme (Hyperhydrierung) und eine niedrige Vitalkapazität. Gewöhnlich verschwinden die Symptome innerhalb von 24 Stunden. Hilfreich sind betaadrenerge Agonisten wie Salbutamol, Diuretika sowie Steroide unter antibiotischer Abschirmung.

US-Forscher verglichen nun zehn Sportler, die schon einmal ein SIPE entwickelt hatten, mit 20 Kontrollpersonen. Alle bekamen Katheter in die A. radialis und in die Pulmonalarterien eingelegt. Auf einem Ergometer belasteten sie sich über 6-7 Minuten in kaltem Wasser. Danach wiesen die SIPE-Probanden signifikant höhere pulmonalarterielle und Wedge-Drücke auf. Als sie die Belastung allerdings 150 Minuten nach der Einnahme von 50 mg Sildenafil wiederholten, lagen sie auf dem Niveau der Kontrollgruppe. Die Autoren sehen hier eine Methode der Risikominimierung.

Wer kein Sildenafil einnehmen will, kann z. B. einen Neoprenanzug mit geringer Kompression anziehen, sich vor Beginn des Wettbewerbs langsam im Wasser akklimatisieren und im Vorfeld nicht extrem viel trinken. Die Veranstalter sollten darauf achten, dass das Wasser nicht zu kalt ist.

Prof. Dr. med. C. Diehm

- Moon RE et al. Swimming-induced pulmonary edema: pathophysiology and risk reduction with sildenafil. Circulation. 2016;133:988-996 